\title{
Finding the Evidence Base Using Citation Networks: Do 300 to 400 US Physicians Die by Suicide Annually?
}

J Gen Intern Med 36(4):1129-31

DOI: $10.1007 / \mathrm{s} 11606-020-05824-\mathrm{Z}$

(c) Society of General Internal Medicine 2020

\section{INTRODUCTION}

An eye-catching estimate of physician suicide incidence is that "300 to 400 US physicians die by suicide annually." It is important that the scientific evidence be sifted from an enormous volume of literature to prevent the propagation of inaccurate claims. ${ }^{1}$ Physician suicide incidence varies depending on method of ascertainment, country, specialty, stage of profession, and gender. ${ }^{2}$ Citation distortion occurs when a claim is stated as fact yet actually has no supportive empiric evidence. ${ }^{3}$ How can the provenance and accuracy of claims regarding physician suicide be traced?

One method deriving from graph theory is to construct a citation network, which represents claims made in published literature and their supporting references, data, and other components. Greenberg previously traced a single scientific claim's lineage, demonstrating citation distortion with successive publications. ${ }^{3}$ A large Alzheimer's disease research community used a similar approach to organize research findings in an annotated knowledge base to facilitate continued scientific discovery about the condition. ${ }^{4}$ In this report, we constructed a claim-specific citation network. ${ }^{3}$ focusing on one claim about physician suicide deaths. This is a proof-ofconcept to capture evidence that can be used to track estimated rates of physician suicide.

\section{METHODS}

Claims were extracted from a subset of articles included in a scoping review of peer-reviewed literature on physician suicide. $^{2}$ The review included a variety of article types published between 1903 and 2018 from 37 countries. The search was updated in August 2019 and additional articles identified, in accordance with the original inclusion criteria of the review. We curated from each article the claim (about the number of physicians who die by suicide annually), claim attribute (author), cited reference(s), and cited data or methods, if provided. Each set of data represents a type of micropublication, ${ }^{5}$ which is a semantic model for scientific claims and evidence that enables knowledge discovery and inference across networks of information. We constructed each micropublication

Received February 15, 2020

Accepted March 26, 2020

Published online May 27, 2020 using extracted data, and then manually combined them into one citation network representing the claims, their relationships, and supporting references and data ${ }^{5}$

\section{RESULTS}

Claims from 26 articles were identified for modeling as micropublications (Fig. 1). ${ }^{5}$ Of 26 articles, 12 (46.2\%) cited no references to support their claim about annual physician suicide deaths. Four articles (15.4\%) claim a different number of annual physician suicide deaths than the claim of interest. The remaining articles cite ten references, of which six make the same claim with no references, three do not make the claim, and one is a website that no longer makes this claim as of August 2019.

The earliest article, published in 1968, reported 228 physician suicides over a 2-year period, based on obituary materials collected by JAMA. ${ }^{6}$ Combined with an estimated 296,000 physicians in the USA at the time, this resulted in a crude annual suicide rate of 38.4 per 100,000 physicians. Reapplying this crude estimate using 2016 census from the Federation of State Medical Boards, 366 physician suicides are estimated annually. However, none of the 26 articles cited this article. Figure 2, based on Greenberg, ${ }^{3}$ shows the manually constructed citation network from collected data.

\section{DISCUSSION}

Findings from tracing the claim lineage about physician suicide rates reveal inadequate data or methods. Clearly cited and trustworthy claims would facilitate a clearer understanding of physician suicide and support further research and design of policies to support physician well-being. Our study is novel because citation networks to trace claim lineage have not been applied to the study of physician suicide. The citation network revealed how easily inadequately supported claims can be propagated in peer-reviewed literature. Advocates, researchers, and the medical community should remain vigilant of potential misinformation or information distortion.

A limitation is that we traced a single verbatim claim. Suicide incidence is often reported as an annual suicide rate per 100,000 persons. These data could be extracted, modeled, and incorporated into this citation network, along with an accompanying census of the number of US physicians for each year, to better clarify the origins of the claim of interest. Furthermore, tracing the types of claim distortion could be informative in future investigation to determine how best to 


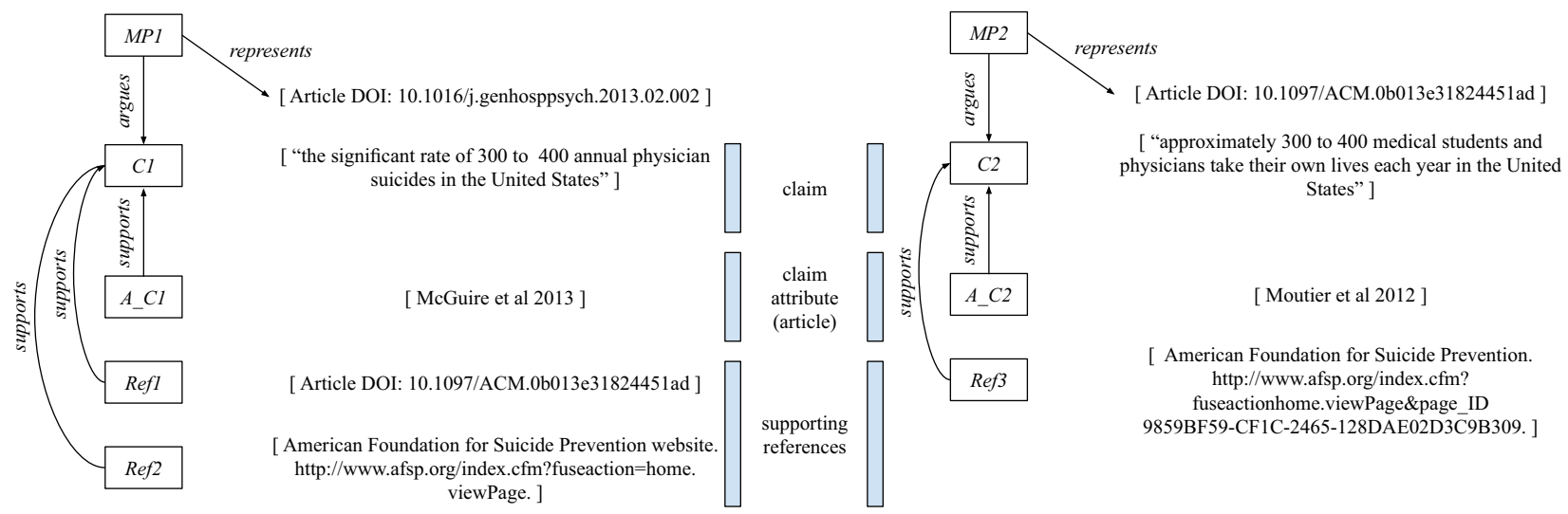

$M P 1, M P 2$ are micropublications; $C 1, C 2$ are claims; $A_{-} C 1, A_{-} C 2$ are attributions (article and its author); Ref1, Ref2, Ref3 are supporting references for each claim. Each relationship (indicated by an arrow) is what is provided by the article (claim attribute); for example, McGuire et al 2013 makes a claim and cites two references as supportive of the claim.

Figure 1 Micropublication model of two articles making a claim about the number of physician suicide annually.

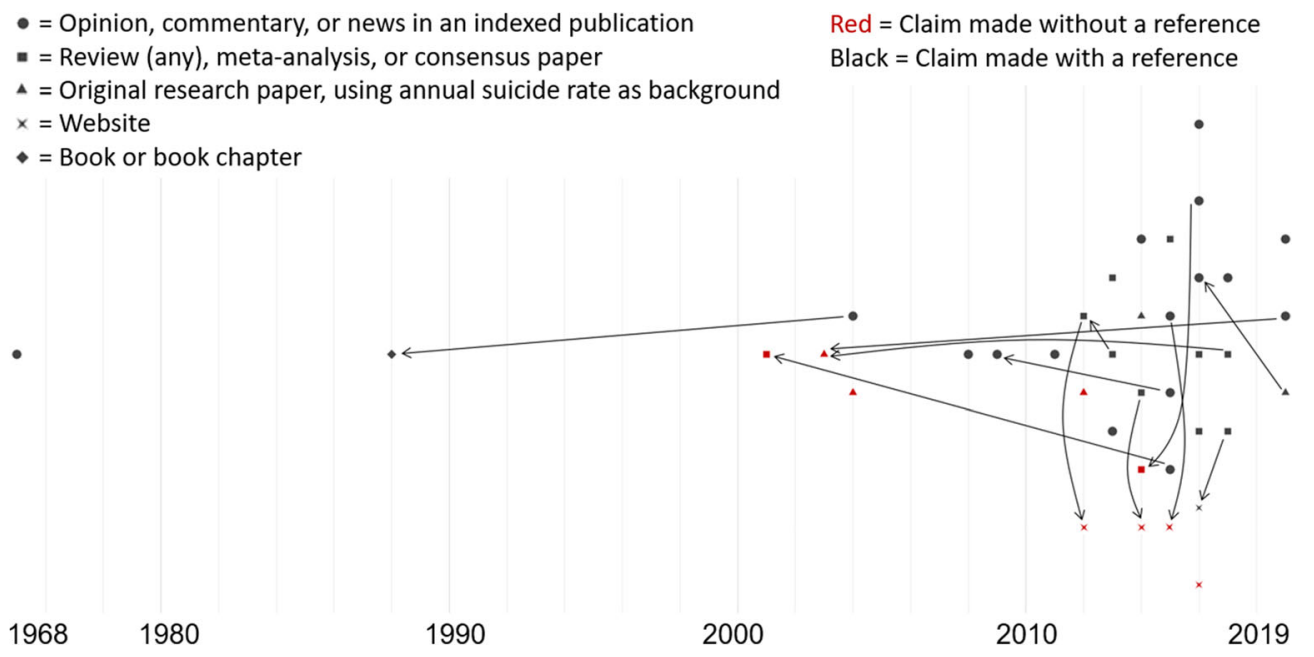

Figure 2 Citation network for multiple articles making one claim about the number of physician suicides annually. The colors are visible in the online version of the article.

mitigate them. Our approach of building citation networks about physician suicide appears promising for future studies and for ensuring preserved accuracy of scientific work in this field.

Tiffany I. Leung, MD, MPH, FACP, FAMIA

Faculty of Health, Medicine and Life Sciences,

Maastricht University ,

Postbus 5800,6202 AZ Maastricht, The Netherlands

Sima Pendharkar, MD, MPH, FACP

Division of Hospital Medicine, The Brooklyn Hospital Center, Icahn School of Medicine Mt. Sinai,

Brooklyn, NY 11206, USA

\section{Chwen-Yuen Angie Chen, MD, FACP, FASAM}

Department of Primary Care and Population Health, Stanford University,

211 Quarry Road, 4th Floor, Hoover Pavilion, Palo Alto, CA 94304, USA

Michel Dumontier, $P h D$

Institute of Data Science,

Maastricht University,

Paul-Henri Spaaklaan 1, 6229 GTMaastricht,

The Netherlands

Corresponding Author: Tiffany I. Leung, MD, MPH, FACP, FAMIA; Faculty of Health, Medicine and Life Sciences, Maastricht University Postbus 5800, 6202 AZ Maastricht, The Netherlands (e-mail: t.leung@maastrichtuniversity.nl). 


\section{Compliance with Ethical Standards:}

Conflict of Interest: The authors declare that they do not have a conflict of interest.

\section{REFERENCES}

1. Clark T. Argument graphs: Literature-Data Integration for Robust and Reproducible Science. In: International Workshop on Capturing Scientific Knowledge. 2015.

2. Leung TI, Chen C-YA, Snyder R, Pendharkar S. Physician Suicide: A Scoping Review. medRxiv 19004465; https://doi.org/10.1101/19004465
3. Greenberg SA. How citation distortions create unfounded authority: analysis of a citation network. BMJ. 2009;339:b2680.

4. Clark T, Kinoshita J. Alzforum and SWAN: the present and future of scientific web communities. Brief Bioinform. 2007;8(3):163-171.

5. Clark T, Ciccarese PN, Goble CA. Micropublications: a semantic model for claims, evidence, arguments and annotations in biomedical communications. J Biomed Semantics. 2014;5:28.

6. Craig AG, Pitts FN. Suicide by physicians. Dis Nerv Syst. 1968;29:763-72.

Publisher's Note: Springer Nature remains neutral with regard to jurisdictional claims in published maps and institutional affiliations. 